\title{
On the reliability of negative heat capacity measurements
}

\author{
M. D’Agostino ${ }^{\text {a }}$, R. Bougault ${ }^{\mathrm{b}}$, F. Gulminelli ${ }^{\mathrm{b}}$, M. Bruno $^{\mathrm{a}}$, \\ F. Cannata ${ }^{\text {a }}$, Ph. Chomaz ${ }^{\mathrm{c}}$, F. Gramegna ${ }^{\mathrm{d}}$, I. Iori ${ }^{\mathrm{e}}$, \\ N. Le Neindre ${ }^{\mathrm{a}}$, G. V. Margagliotti ${ }^{\mathrm{f}}$, A. Moroni, ${ }^{\mathrm{e}}$, G. Vannini ${ }^{\mathrm{a}}$. \\ ${ }^{a}$ Dipartimento di Fisica and INFN, Bologna, Italy \\ ${ }^{\mathrm{b}}$ LPC Caen (IN2P3-CNRS/ISMRA) et Université F-14050 Caen Cédex, France \\ ${ }^{\mathrm{c}}$ GANIL (DSM-CEA/IN2P3-CNRS), B.P.5027, F-14021 Caen Cédex, France \\ d INFN Laboratorio Nazionale di Legnaro, Italy \\ e Dipartimento di Fisica and INFN, Milano, Italy \\ ${ }^{\mathrm{f}}$ Dipartimento di Fisica and INFN, Trieste, Italy
}

\begin{abstract}
A global protocol for the thermostatistical analysis of hot nuclear sources is discussed. Within our method of minimization of variances we show that the abnormal kinetic energy fluctuation signal recently reported in different experimental data $[1,2]$ is a genuine signal of a first order phase transition in a finite system.
\end{abstract}

PACS: 25.70.pq; 64.70.-p; 65.50.+m; 64.60.Fr; 24.10.Pa, 24.60.-k.

Key words: Liquid-gas phase transition; fluctuations; nuclear heat capacity; multifragment emission.

\section{Introduction}

The existence of systems exhibiting a negative heat capacity has been postulated in the seventies in the context of collapsing self-gravitating systems [3]. The physical origin of the apparent paradox of an object cooling while absorbing thermal energy is the impossibility of defining a thermodynamical limit for systems interacting via non saturating forces. A similar situation occurs in mesoscopic systems where the range of the interaction, though short, is comparable to the linear dimension of the system [4]. In all these situations the appearance of a negative heat capacity branch for isolated microcanonical 
systems is predicted by theory as a particular example of the specific character of first order phase transitions in finite systems [5].

From the experimental point of view, the first evidences of a negative heat capacity have been reported very recently $[1,2,6]$. In the Haberland experiment [6] small sodium clusters have been demonstrated to show a negative heat capacity in the region of the solid to liquid transition, through the measurement of curvature anomalies in the energy distribution near the transition temperature. In the case of the liquid to gas like phase transition of hot nuclear sources $[1,2]$ negative heat capacities have been observed via the study of kinetic energy fluctuations [7]. In the MULTICS-MINIBALL experiment an isotropic source of approximately 200 particles has been selected in peripheral and semi-peripheral collisions of $\mathrm{Au}$ nuclei impinging on a $\mathrm{Au}$ target at $35 \mathrm{MeV}$ per nucleon $[1,8]$. In the INDRA experiment quasi-fusion isotropic sources of approximately the same size have been extracted in $X e+S n$ collisions with a beam energy varying from 32 to $50 \mathrm{MeV}$ per nucleon [2,9]. The two data samples involving different reaction mechanisms and measured with different experimental devices give a negative heat capacity signal in the same excitation energy range. These measurements not only represent a very strong evidence of the expected liquid to gas phase transition of nuclear matter [10], but also show that nuclear thermodynamics can start to be addressed in a quantitative way.

A clear evaluation of the degree of reliability of these results is therefore of a prime interest for the understanding of the nuclear equation of state as well as the general topic of phase transitions in finite systems. This is not trivial, since the measurement of kinetic energy fluctuations is confronted with many technical as well as conceptual difficulties.

First, a very careful analysis has to be performed in order to select an equilibrated or close to equilibrium source from the highly dynamical process of heavy ion collisions.

Then, in order to perform the fluctuation analysis, the energy deposited in the system should be fully measured on an event by event basis. This ideal situation is never realized in an actual experiment and one has to check that the limitations and inefficiencies of the experimental devices do not distort the expected signal.

Finally secondary evaporation has to be deconvoluted from the asymptotically detected partitions, in order to study the correct energy balance at freeze out. This can only be done via working hypotheses that have to be carefully scrutinized and eventually constrained by experimental results.

In this paper we want to analyze in detail all the possible sources of uncertainty in the heat capacity analysis, coming from the missing information of data. 
We wish to establish a general protocol for a thermostatistical analysis of nuclear sources that allows to deal with mean values as well as higher order moments of any observable within a minimum bias technique. The analysis will here be specialized to heat capacity measurements, but the problems we will be confronted with are common to all high order moment analysis of multiparametric data [11]. We will demonstrate that within this general protocol the negative heat capacity signal cannot be artificially generated by the bias of the analysis. We show that the location of the coexistence zone in the nuclear phase diagram and the value of the latent heat can be approximately evaluated but they are still subject to large uncertainties due to the limitations of the present detection devices.

The plan of the paper is as follows: in section 2 the principles of the fluctuation analysis and the present experimental status are summarized. In section 3 the fluctuation technique is applied to some well known models of nuclear fragmentation. Section 4 briefly addresses the problem of the correct sorting variables to perform a thermostatistical analysis and the effect of binning on observables. In section 5 the general method of dealing with a missing information in order to minimize the bias of the analysis is presented. This method is specialized in the two following sections to the reconstruction of primary partitions (section 6) and to the distortions induced by the imperfect calorimetry (section 7). In section 8 the physical parameters entering the analysis are discussed in detail and some methods to constrain the value of these parameters with information coming from the experimental data are proposed. Finally section 9 contains conclusions and outlooks.

\section{Kinetic energy fluctuations and microcanonical heat capacity}

First order phase transitions in finite systems are univocally defined by the abnormal convexity of the thermodynamical potential $[4,5]$ in the state variables plane. For a microcanonical system undergoing a liquid gas phase transition, this anomaly produces a characteristic behaviour of the heat capacity: a negative branch delimited by two divergences that define the crossing of the coexistence line. This behaviour is suppressed only if sharp boundary conditions are imposed on the system, i.e. if volume can be considered as the relevant state variable of the microcanonical statistical ensemble [12]. This is certainly not the case for nuclear sources which are open systems not constrained by any boundary condition. In the experimental situation the volume is rather an observable known at best in average and impossible to use as a sorting variable. In such a case the pressure, interpreted as the Lagrange multiplier associated to the volume, appears to be the relevant state variable together with the total deposited energy; a convex intruder is expected and the energy fluctuations (see below) are related to $C_{p}$. 
In Ref. [7] it was proposed that the microcanonical heat capacity can be measured using partial energy fluctuations. For a classical system with momentum independent interactions the total energy $E$ can be decomposed into two independent components, its kinetic $\left(E_{k}\right)$ and interaction energy $\left(E_{I}\right)$. Since the energy partition directly depends on the partial entropies $S_{k}$ and $S_{I}$, the kinetic energy variance can be related, in the Gaussian approximation, to the heat capacities $[7,13]$ :

$$
A_{0} \sigma_{k}^{2} \simeq T^{2} \frac{c_{k} c_{I}}{c_{k}+c_{I}}
$$

where $c_{k}$ and $c_{I}$ are the kinetic and interaction microcanonical heat capacities per particle calculated for the most probable energy partition characterized by a microcanonical temperature $T$. Equation (1) can be inverted to extract, from the observed fluctuations, an estimate of the heat capacity [7]:

$$
\frac{C}{A_{0}}=c \simeq c_{k}+c_{I} \simeq \frac{c_{k}^{2}}{c_{k}-A_{0} \sigma_{k}^{2} / T^{2}}
$$

From eq.(2) we can see that the heat capacity becomes negative if the kinetic energy fluctuations overcome the canonical expectation $A_{0} \sigma_{k}^{2} / T^{2}=c_{k}$. It is amazing that the constraint of energy conservation leads in the phase transition region to larger fluctuations than in the canonical case where the total energy is free to fluctuate. This is because the kinetic energy part is forced to share the total available energy with the interaction part. When the interaction part presents a negative heat capacity, the jumps from liquid to gas induce strong fluctuations in the energy partitioning. It is also interesting to remark that if the kinetic equation of state is known, the most probable value of the kinetic energy, as well as the average one, acts as a very powerful microcanonical thermometer [7].

In principle one could argue that the same information on the heat capacity can be obtained by taking the derivative of the correlation between the temperature and the excitation energy (the so called caloric curve). We want to stress that fluctuations are characteristic of the state and so depend on the pertinent state variable while the caloric curve $T(E)$ depends upon the specific thermodynamical transformation from one state to another. Therefore, the information obtained by taking the derivative of the measured caloric curve may differ from the information coming from the fluctuations [14].

In order to perform a thermostatistical analysis one has to collect a data sample which corresponds to a (collection of) microcanonical ensembles. The microcanonical ensemble is relevant for the analysis of experimental data because of the absence of a heat bath and since using calorimetry techniques the excitation energy can be measured on an event-by-event basis. For any 
arbitrary shape of the excitation energy distribution the events can thus be sorted in constant energy bins, i.e. in microcanonical ensembles.

Single source complete events have to be selected with a constant value for the collected charge in each energy bin. In both analyses only well detected events (total detected charge larger than 70\% [1] or 80\% [2] of the Au charge) were considered. After a shape analysis [15] central collisions are isolated from the $X e+S n$ collisions with a selection on the flow angle $\Theta_{F}$ between the beam axis and the main eigenvector of the kinetic energy tensor $\left(\Theta_{F}>60^{\circ}\right)$. For the $A u+A u$ system, peripheral collisions of a predominantly binary character are selected by requiring the velocity of the largest fragment in each event to be at least $75 \%$ of the beam velocity. Then fragments of each event are considered as originated by the quasi-projectile if forward emitted in the ellipsoid reference frame. Moreover in both data sets only events where the total reconstructed source charge results within $10 \%$ of the $A u$ charge are kept. For details about the selection criteria, see $[1,2]$ and the references quoted therein. For both reactions the selected events are close to the maximum of the total charge distribution. As it will explained below in more detail, this means that source mass fluctuations are under control and that we are dealing with a statistically significant sample of each centrality (i.e. excitation energy) bin.

To check the quality of the source selection criteria, a standard procedure consists in verifying that events are spherically symmetric in momentum space. Of course it is very likely that in the dynamical preparation of the nuclear source the shape degree of freedom may not be completely relaxed; such a case could in principle be addressed within a statistical ensemble where deformation is explicitly accounted for. However restricting the analysis to spherical systems guarantees that non statistical effects, as preequilibrium or midrapidity emission, do not pollute the statistical sample.

In the case of central collisions the isotropic character of fragment emission is implicit in the flow angle selection and has been verified in detail comparing to simulations $[9,16]$. Concerning the quasi-projectile (QP) events, the charge density [17] of selected events is plotted in Figure 1 in different excitation energy bins. The QP fragments are normally distributed even for the less peripheral collisions. Moreover the backward emitted fragments in the ellipsoid reference frame (which are excluded in the subsequent analysis) are perfectly consistent with the filtered simulation of a quasi-target source symmetric to the QP. This suggests that midrapidity emission of fragments is negligible for the selected events (see Fig.s $1 \mathrm{f}$ ) and 3 of Ref. [8]), at variance with other experimental studies [18], where different entrance channels or selection criteria enhance a dynamical emission at semi-peripheral impact parameters.

More delicate is the contribution of light particles $(Z=1,2)$ which are likely to be emitted during the whole collision process and not confined to the freeze 


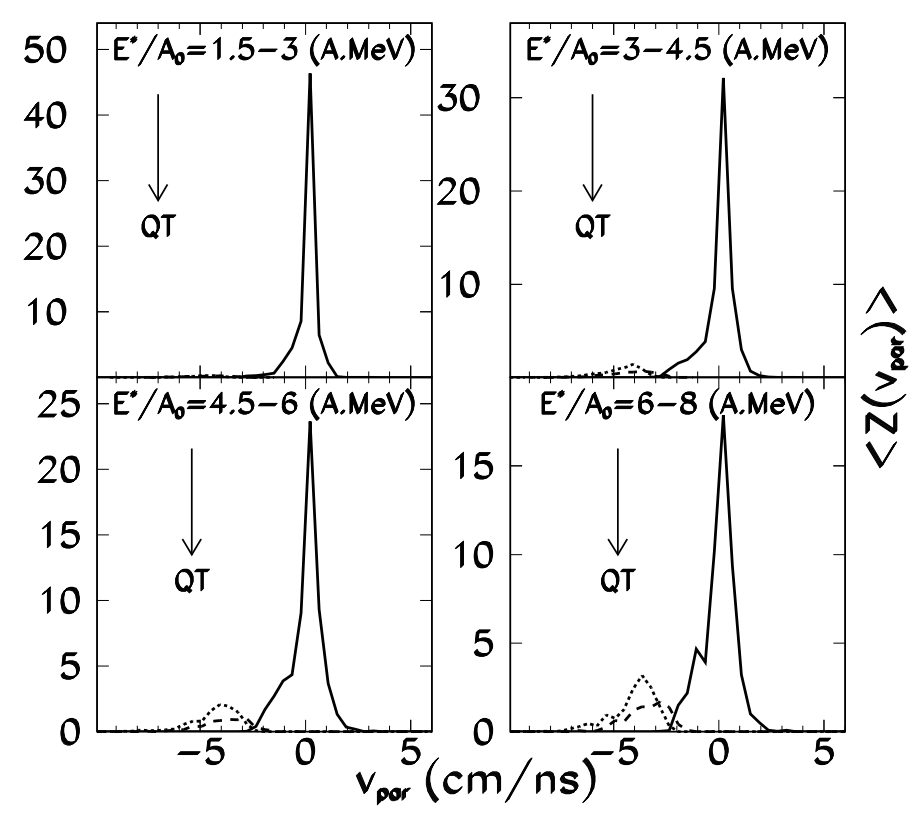

Fig. 1. Detected average fragment charge ( $Z \geq 3$ ) for the peripheral $A u+A u$ collisions as a function of the parallel velocity in the quasi-projectile reference frame, for different excitation energy bins. Full lines: fragments belonging to the quasi-projectile. Dashed lines: fragments rejected from the quasi-projectile selection. Dotted lines: simulated and filtered quasi-target contribution.

out stage. For the $X e+S n$ central collisions, in order to avoid the possible contamination of preequilibrium, only particles emitted between $60^{\circ}$ and $120^{\circ}$ in the center of mass are considered and their contribution is doubled to compensate for the forward/backward anisotropy [2]. In the same way for the $\mathrm{Au}$ quasi-projectile, a possible contribution of midrapidity emission is minimized by substituting the backward light particle emission by the symmetric of the forward emission, in the quasi-projectile reference frame [1].

The excitation energy of the source is reconstructed from calorimetry on an event by event basis [8,9]. Different hypotheses have been considered about the number and energy of the undetected neutrons [8] and will be discussed in detail in the following.

The relevance of the thermostatistical analysis depends on the approximation at which an equilibrium is realized. Equilibrium, being by definition the reducibility of the multidimensional information to a number of macroscopic constraints, can never be proved but only be disproved, even for an isotropic source. As a general statement, the degree of approximation of an equilibrium is indicated by the degree of the agreement of data with the prediction of a 
statistical model containing the same constraints as the data. For both data sets discussed in this paper $[2,8,9,19]$ a very good agreement has been found with the predictions of the statistical model SMM [20] for static as well as dynamical variables. In particular a detailed analysis of the average kinetic energy of the fragments indicates the presence of a collective component ranging from $0 \mathrm{~A} . \mathrm{MeV}$ for the most peripheral collisions of the $A u+A u$ system to $2 \mathrm{~A} . \mathrm{MeV}$ for the central $\mathrm{Xe}+\mathrm{Sn}$ ones at $50 \mathrm{~A} . \mathrm{MeV}$ incident energy. The good model reproduction of several observables suggests that, in this energy range, the collective motion can be to a good approximation superimposed to a thermal picture. The collective contribution to the excitation energy has been removed in the subsequent analysis.

Let us now turn to the heat capacity measurement. In order to extract the heat capacity from eq. (2) one has to decompose the total measured excitation energy into the kinetic and interaction contributions at the time of fragment formation, i.e. at freeze out. In the case of nuclear fragmentation data, this is complicated by the fact that the fragments are detected at infinity, after secondary de-excitation, i.e. with lower masses. Moreover, because of the presence of the long range Coulomb interaction, asymptotic kinetic energies have to be corrected for the Coulomb boost. To take into account these distortions, the kinetic energy at freeze out is reconstructed by applying the energy balance event by event:

$$
E_{k}=m_{0}+E^{*}-\sum_{i=1}^{M} m_{i}-E_{\text {coul }}\left(V_{F O}\right)=E^{*}-E_{I}
$$

where $m_{0}, m_{i}$ are the mass excess of the source and of the primary products respectively, $E^{*}$ the excitation energy measured via calorimetry, and $E_{\text {coul }}$ is the Coulomb energy of the partition. The two important unknown quantities here are the primary multiplicity $M$ entering the $Q$-value and the freeze-out volume $\left(V_{F O}\right)$ determining the Coulomb energy. Only qualitative information about these quantities are given by theory. Dynamical models predict a sudden increase of density fluctuations leading to fragment formation at low density, but the actual value of the volume depends on the model. Concerning secondary cooling, a correct description of the temperature dependent partition function is needed to reconstruct the de-excitation chain and discriminate between prompt and secondary emission. At low excitation energies this aim is accomplished by the theory of compound nucleus. At higher excitation energies the opening of multifragmentation channels makes an exact evaluation increasingly difficult. Sophisticated Monte-Carlo calculations of the multifragmentation pattern are available since many years $[4,20,21]$, but the internal partition function at high temperature is poorly known, and the suppression procedure of the state-density integral at high excitation energy is not unique.

The Coulomb energy has been evaluated in Ref.s $[1,2]$ by randomly positioning 
non overlapping spherical primary products in a freeze-out volume from 3 to 6 times larger than the normal volume. The primary multiplicity and the primary masses have been obtained [8] by sharing the final light particles and neutrons among the detected fragments, following two extreme freeze-out hypotheses $[4,20]$. A lower limit for the primary multiplicity can be obtained by assuming (hot fragment hypothesis) that the totality of light charged particles and neutrons is emitted by the hot primary fragments in a secondary decay process. An upper limit (cold fragment hypothesis) consists in assuming that all light charged particles are primary and de-excitation concerns only neutron evaporation.

To apply eq. (2) we also need to calculate the microcanonical temperature $T$ of the system. An estimator of $T$ can be obtained by inverting the kinetic equation of state

$$
\left\langle E_{k}\right\rangle=\left\langle\sum_{i=1}^{M} a_{i}\right\rangle T^{2}+\left\langle\frac{3}{2}(M-1)\right\rangle T
$$

where the brackets $<\cdot>$ indicate the average on the events with the same $E^{*}$ and $a_{i}$ is the level density parameter [8]. The temperatures of the system obtained in the two freeze-out hypotheses can be considered as an upper and a lower limit of the actual freeze-out temperature [8].

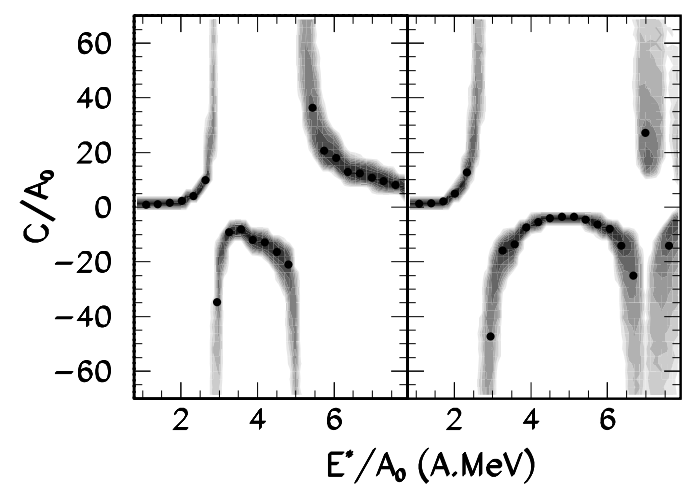

Fig. 2. Heat capacity per nucleon (solid symbols) obtained from equation (2) for the $Q P$ data. The panel on the left corresponds to hot primary fragment in a freeze-out volume $3 V_{0}$. The panel on the right corresponds to cold primary fragments in a freeze-out volume $6 V_{0}$. The grey contour indicates the confidence region for $C / A_{0}$.

The functional form of eq.(4) is certainly a reasonable ansatz for the average kinetic energy of an ensemble of fragments, but an extra source of uncertainty 
comes from the value chosen for the level density parameter. This will be discussed in Section 8.

Figs. 2 and 3 show the final result for the total heat capacity obtained from the Au quasi-projectile and the central $X e+S n$ collisions $[1,2] . c_{k}$ in eq.(2)
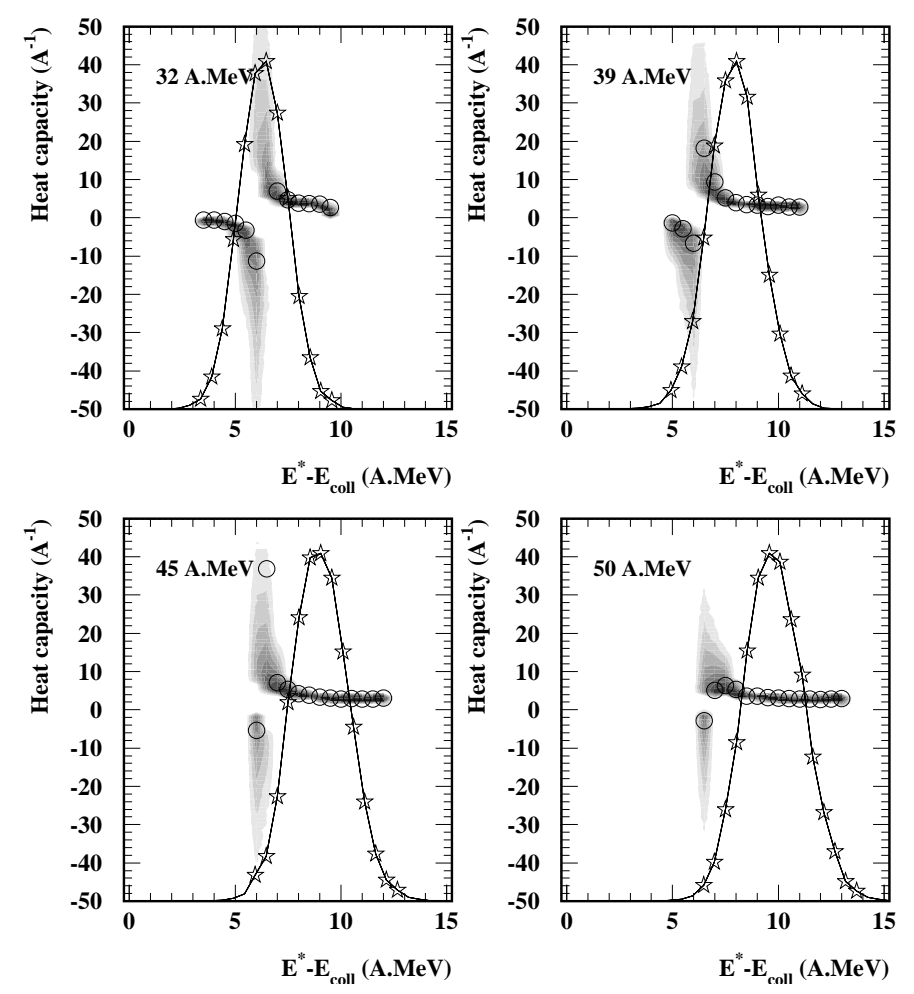

Fig. 3. Specific heat capacity (open points) obtained from eq. (2) for the central $X e+S n$ data at four different bombarding energies within the hot fragment hypothesis as calculated in ref. [2]. Lines and stars: calorimetric excitation energy distributions with collective flow correction. The grey contour indicates the confidence region for $C / A_{0}$.

has been obtained by taking the numerical derivative of $\left\langle E_{k}\right\rangle$ with respect to $T$. The grey contour represents the $C$ distribution, evaluated through a Monte-Carlo error simulation program. Only statistical errors are taken into account in the evaluation of this confidence region. A distinct negative branch appears pointing to a 1-st order liquid-gas phase transition, the distance between the poles being associated with the latent heat. A wide range of impact parameters, leading to a widely spread excitation function, is available for the quasi-projectile data, allowing to follow the whole behavior of the heat capacity in the pure as well as in the mixed phases. On the other side in the central collisions sample the selection of the quasi-fused source isolates very central impact parameters and the heat capacity can be measured only around the transition on the vapor side. In this energy interval the QP data are subject to some uncertainties due to the lack of statistics and the difficulties in the source selection, however the agreement between the two sets of data is remarkable. 
A negative heat capacity branch appears in the same excitation energy range in these different collisions leading to different reaction mechanisms, detected with different experimental devices, selected and analyzed with independent methods.

A word of caution is however necessary. Negative heat capacity is obtained when kinetic energy fluctuations exceed the value of the kinetic heat capacity $c_{k}$ (see eq.(2)). $c_{k}$ itself has an upper bound given by the classical Boltzmann limit $c_{k} \leq 3 / 2$. One may wonder if the inefficiencies of the apparatus and the reconstruction hypotheses may introduce an uncontrolled source of fluctuations that may mock up the negative heat capacity signal. In the following sections we will address in great detail all the possible sources of uncertainty related to the fluctuation analysis.

\section{Can we measure a positive heat capacity?}

A first global check of the experimental method consists in applying the fluctuation analysis to a sample of theoretical events generated with statistical models. Two well known and sophisticated models, often used to simulate heavy ion reaction data, are SMM [20] and GEMINI [22]. SMM aims to describe multifragmentation at low freeze out density and for the typical volumes used (around three times the normal source volume) shows a first order liquid-gas like phase transition [23]. GEMINI describes fragment production as a sequence of binary fission-like emissions at a density close to the normal nuclear matter density. The thermodynamics of this model has, to our knowledge, never been scrutinized. Because of the hypothesis of low emission rate (i.e. low vapor-like pressure) and high density we expect that the model should be close for all excitation energies to the coexistence line on the liquid border, and it should not show negative heat capacity. We notice incidentally that the data we are discussing are very well reproduced by SMM at all excitation energies $[8,9]$ while the GEMINI code can only reproduce the most peripheral QP data.

The procedure sketched in the previous section has been applied to simulated SMM and GEMINI data in the energy range between 1 and $9 \mathrm{~A} . \mathrm{MeV}$. In both cases the freeze out has been reconstructed within the hot fragment hypothesis [1]. Figure 4 shows the resulting kinetic energy fluctuations as well as the corresponding heat capacity. The normalized fluctuations show a peak in the SMM data while they are monotonically decreasing in the case of the GEMINI model. This tends to confirm the claim [7] that a peak in the reduced kinetic energy variance is by itself a signature of a thermodynamical phase transition. If in addition the temperature is estimated via eq.(4), it is possible to convert the fluctuation signal into a heat capacity. Abnormal fluctuations 


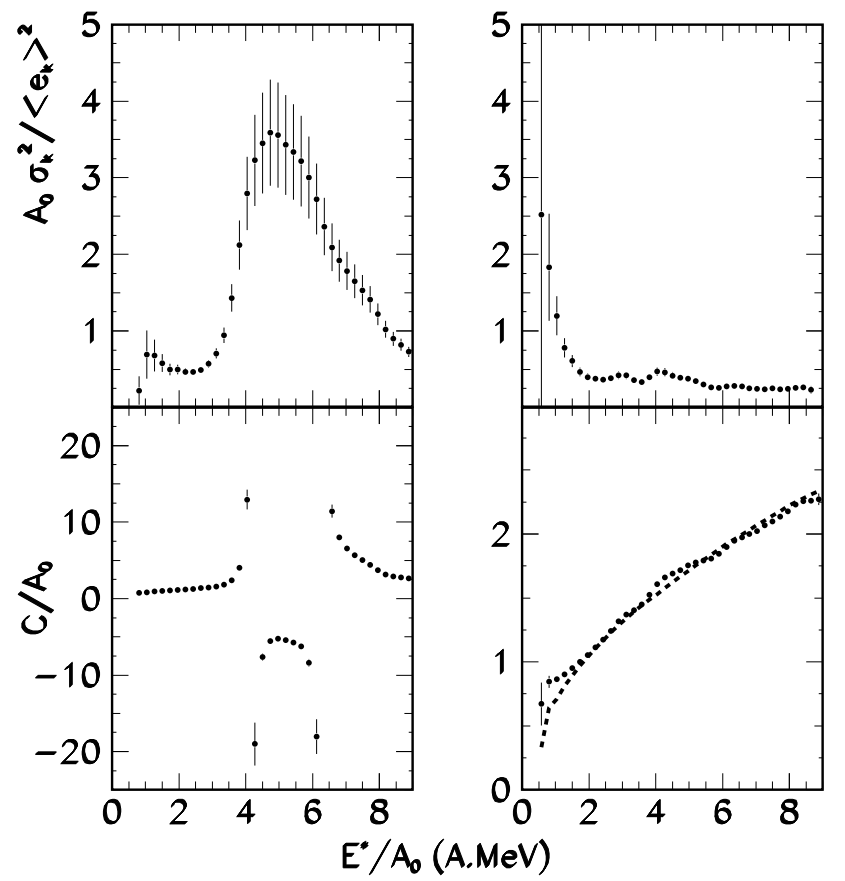

Fig. 4. Normalized kinetic energy fluctuations (upper part) and the corresponding reconstructed heat capacity (lower part) for SMM (left) and GEMINI (right) simulations as a function of the excitation energy. Dashed line: numerical derivative of the theoretical caloric curve.

are obtained for the SMM simulation while the heat capacity for GEMINI is a monotonically increasing function of the excitation energy. It is interesting to remark that for the GEMINI calculation the reconstructed heat capacity is very close to the derivative of the caloric curve (dashed line in figure 4) even if the freeze out reconstruction is certainly not suited to a sequential decay scenario. This can be understood since in a sequential scenario a relatively low number of channels is open. The fluctuations of the interaction energy $E_{I}$ are then very small and eq.(2) gives a heat capacity close to the kinetic heat capacity. For the same reason the functional dependence on temperature of the reconstructed kinetic energy is similar to the total caloric curve. In addition eq. (4) appears to give a reasonable estimate of the temperature of the model, the integration over the deexcitation chain being only a minor correction. This result seems to indicate that eq.(2) is a powerful tool to extract heat capacities in presence or in absence of a phase transition, for sequential as well as simultaneous emission processes. 


\section{The effect of binning}

A very first concern when applying a microcanonical analysis to a set of (experimental or simulated) data is the effect of event mixing due to binning. Equation (2) is derived under the microcanonical constraint, i.e. a strict conservation of energy and mass. The process of energy binning violates energy conservation while the dynamical fluctuations of the entrance channel and the lack of detection of neutrons and fragment masses induce fluctuations in the source size in each excitation energy bin, which is controlled at the 10-20\% level only $[1,2]$.

The effect of varying the width of the energy bins is explored in the left part of figure 5 using a SMM simulation. 70000 events are generated from a continuous and flat distribution of excitation energies in the range 1-10 A.MeV for an Au source. This statistical sample will be also used for all the successive analysis presented in this paper. In all cases the freeze out is reconstructed, within the hot fragments hypothesis, from the simulated final partitions as in the experimental data (see section 2). To isolate the genuine effect of binning, the exact input excitation energy of the model is used in each event. Additional distortions due to the deficiencies of the experimental calorimetry will be discussed in section 7 .

From figure 5 one can see that the average values of the presented observables are too smoothly varying to be affected even by a non realistic binning as wide as $\Delta E=0.9 \mathrm{~A} . \mathrm{MeV}$. When the width of the bin is increased, fluctuations are smoothed out but the height of the maximum is not affected. This is easy to understand since any (small) violation of the energy conservation perturbs the microcanonical constraint $E^{*}=E_{k}+E_{I}$. This flattens the normalized fluctuation $\sigma_{k}^{2} /\left\langle e_{k}\right\rangle^{2}$ similarly to the fluctuation suppression operated by the microcanonical event mixing in the canonical ensemble. The effect is barely visible for bin widths smaller than about $1 \mathrm{~A} . \mathrm{MeV}$. A similar effect is obtained if a (fluctuating) non thermal component is added to the excitation energy [2] to simulate the effect of an imperfect subtraction of the collective flow in central collisions. The same analysis applied to the data shows no sizeable effect due to the bin width [24]. The fluctuation smoothing is barely visible because the calorimetric uncertainties cannot be disentangled from the effect of binning (see next sections).

Next we turn to the possible spurious fluctuations induced by violations of mass conservation. As it is done with the data, only the light particles emitted in one half of the solid angle in the SMM simulation are kept and their contribution is symmetrized backward. Once again we leave the effect of this doubling procedure on the calorimetric reconstruction of excitation energy to section 7, and use here the input energy of the model. The doubling of light 


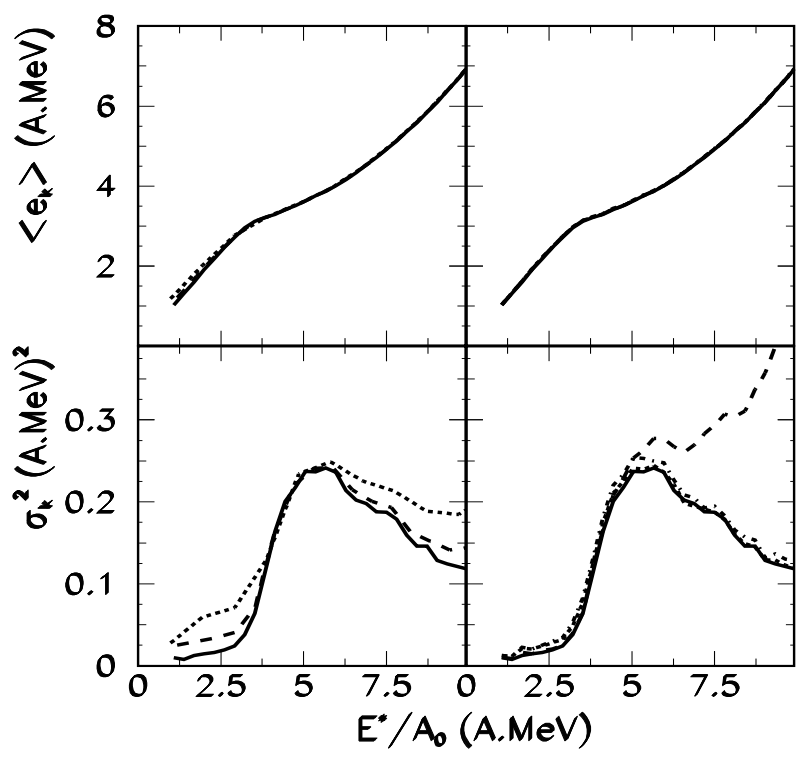

Fig. 5. First (upper part) and second moment (lower part) of the SMM kinetic energy distribution reconstructed within the hot fragment hypothesis [1]. Left part: different energy bin widths $\Delta\left(E^{*} / A_{0}\right)=0.3$ (full line), 0.6 (dashed line), $0.9 \mathrm{~A} . \mathrm{MeV}$ (dotted line). Right part: effect of the non conservation of mass induced by the doubling of light particles (see text).

particles leads to mass number fluctuations that increase with the excitation energy and that are comparable to the experimental mass width in each excitation energy bin. The right part of figure 5 shows that this mass fluctuation does not affect average quantities while a strong deformation of the original fluctuation signal (full line) is apparent if we calculate the variance of the total kinetic energy (dashed line). If energies per nucleon are used (dash-dotted line) the signal is almost not perturbed. If events of mass number differing more than $10 \%$ from the original source mass are rejected (dotted line) the initial fluctuations are also approximately restored. Both procedures are used for the analysis of the data. Since these general results do not depend on the details of the simulation employed, we can be confident that no spurious fluctuations are generated either by the procedure of light particles doubling or by the $10 \%$ fluctuations of the source size.

\section{$5 \quad$ Dealing with missing information}

Deriving nuclear thermodynamics from the fragmentation sample on an event by event basis, one is systematically confronted with the problem of missing information. This concerns detection limitations (neither neutrons nor frag- 
ment masses are measured, the response of the experimental filter deforms the events) as well as the uncertainties of the freeze out reconstruction (the primary masses, multiplicities and freeze out volume are not known) and the unknown physical parameters (the kinetic equation of state eq.(4) is only an ansatz, and in particular the level density parameter is poorly known at high excitation energy). Since we are interested in first as well as second order moments, the missing information has to be implemented event by event. In principle one would like to restore the missing information in such a way that mean values as well as variances agree with values obtained for the same observables from independent measurements. This is however in general a very tough task. As an example one may be able to measure an average value for the size of the fragmenting source through correlation techniques, but this method does not give an event by event response. Only sophisticated backtracing procedures [25], working in the multivariate space of the source characteristics, provide event by event estimates. However, the obtained distributions turn out fully model dependent.

Here we propose a less ambitious way of dealing with missing information. The heat capacity measurement results from the simultaneous evaluation of partial energy $e_{k}$ fluctuations, total deposited energy and temperature. Heat capacity is negative if the variance $A_{0} \sigma_{k}^{2}$ exceeds a value $T^{2} c_{k}=T^{2} \frac{d<e_{k}>}{d T}$ determined solely by the same partial energy mean value $\left\langle e_{k}>\right.$ through its equation of state $T\left(e_{k}\right)$. Let us suppose that the mean value of the missing observables is known from theory or from an independent measurement. Then the event by event value of the same observables can be fixed to its mean value, such as to systematically minimize the partial energy fluctuations. If then the negative heat capacity signal survives, this cannot be attributed to spurious fluctuation due to not measured quantities. It is important to stress that this conservative attitude guarantees the physical meaning of abnormal partial energy fluctuations but prevents a quantitative analysis of the phase transition, in particular a precise evaluation of the latent heat.

The partial energy $e_{k}$ (i.e. $E_{k} / A_{0}$ ) is obtained as the difference between the total deposited calorimetric energy and the interaction energy at freeze out (eq.(3)). The general conservative philosophy of suppressing fluctuations of not measured quantities is applied in the next section to the missing observables that enter in the interaction energy, and in section 7 to the calorimetry. The average values of not measured observables will be then fixed from independent experimental constraints in section 8 . 


\section{The freeze out reconstruction}

Let us take again SMM as an event generator. The freeze out temperature and kinetic energy variance of the model are shown as thick solid lines in figure 6. In this calculation a constant volume $V=3 V_{0}$ is used. The advantage of dealing with simulated data is that we can study the effect of the interaction energy reconstruction on $E_{k}$, independent of the calorimetry errors. The excitation energy in figure 6 is the input excitation energy of the model. In such a situation the only parameters left in eq.(3) are the freeze out observables, namely the volume entering the Coulomb energy and the primary masses and multiplicity entering the Coulomb term as well as the Q-value. We can take the asymptotic simulated partitions and fix on average these parameters such as to reproduce the theoretical average kinetic energy at freeze out. The way this average information is implemented event by event implies an enhancement or a suppression of the second moment. This can be predicted a priori and does not depend on the specific model that generates the events, used in this paper only for illustration.

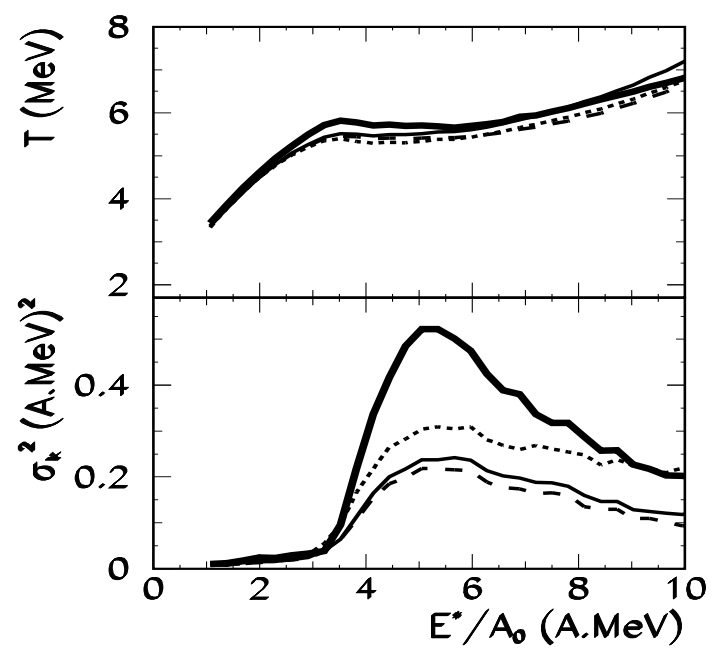

Fig. 6. Temperature (upper part) and kinetic energy variance (lower part) of SMM (thick solid lines) compared to the estimate from the different freeze out reconstructions (see text).

The thin solid line corresponds to the hot fragment hypothesis [1]. All light charged particles and neutrons in each event are shared among the fragments such that they get the same $N / Z$ of the source. (The same result is obtained if, to evaluate the masses of primary fragments, we use a functional $A\left(Z, E^{*}\right)$ fitted to SMM primary fragments). The average freeze out multiplicity is thus 
minimized. Since a deterministic algorithm is used to construct the freeze out multiplicity, the event by event difference between the asymptotic and freeze out multiplicities is suppressed. Not surprisingly, the resulting interaction energy variance strongly decreases. This schematic reconstruction hypothesis was used on experimental data to represent a lower limit to the average freeze-out multiplicity.

In the framework of SMM, this hypothesis is not very realistic, a number of light charged particles being present at the freeze out stage. If we allow an excitation energy dependent percentage of primary light particles (dashed lines), the mean value is better reproduced especially at high energies, but the variance is not affected. Whatever deterministic algorithm we can choose to reconstruct the primary multiplicity, it will imply a suppression of fluctuations if it is implemented on an event by event basis. This is due to the fact that the multiplicity is positively correlated both to the Coulomb potential $V_{c}$ and to the $Q$-value; a reduction in the multiplicity fluctuation will therefore suppress the variance of $V_{c}$ and $Q$ as well as their (positive) covariance. This is why the increase of $\sigma_{k}$ is negligible, even if we impose a large average value of the multiplicity (within the constraint of an approximate reproduction of $<e_{k}>$ ).

On the other hand, any hypothesis that assigns to a missing information a value fluctuating event by event has the effect of increasing $\sigma_{k}$. As an example, the dotted lines in figure 6 are obtained by assuming a flat distribution of freeze-out volumes with a width equal to the average value. The (moderate) increase of $\sigma_{k}$ indicates that, if in the fragmentation data the freeze out volume does fluctuate event by event [14], our analysis, which assumes a constant volume in each excitation energy bin, once again underestimates the physical fluctuations. In all the cases depicted in figure 6 the same level density parameter as in the model [26] has been used; as a consequence the degree of reproduction of $\left\langle e_{k}>\right.$ and $T$ is comparable.

\section{The uncertainties induced by calorimetry}

In the experimental evaluation of the kinetic energy at freeze out (eq.(3)) the total excitation energy is not a fixed external parameter as we have assumed in the previous sections, but it is the result of an event by event calorimetric measurement via

$$
E^{*}=\sum_{i=1}^{N_{c}}\left(m_{i}+E_{i}\right)+N_{n}\left(m_{n}+<E_{n}>\right)-m_{0}
$$

Here $N_{c}$ and $N_{n}$ are the charged particles and neutron multiplicities, $E_{i}\left(E_{n}\right)$ are the kinetic energies, $m_{i}\left(m_{n}\right)$ are the mass excesses of the charged reaction 
products (neutrons) and $m_{0}$ is the mass excess of the source. The calorimetric measurement is affected by a number of uncertainties. The neutron number as well as the neutron energies are not measured; an isotopic resolution is achieved only for light fragments; the experimental filter may deform the energy response though this effect is minimized restricting to only quasi complete events (see section 2). Moreover for the analysis presented here only the light particles emitted in one half of the total solid angle are kept and their contribution is symmetrized to the other half.

To complete this missing information a mass has been assumed for all the detected fragments following the EPAX [27] parameterization; the number of neutrons is then deduced from mass conservation by assuming that the fragmenting source has the same isospin ratio as the composite system (for central events) or as the projectile (for quasi-projectile events). The average neutron energy has been obtained for each excitation energy bin from the total detected kinetic energy, by means of an effective temperature [28].

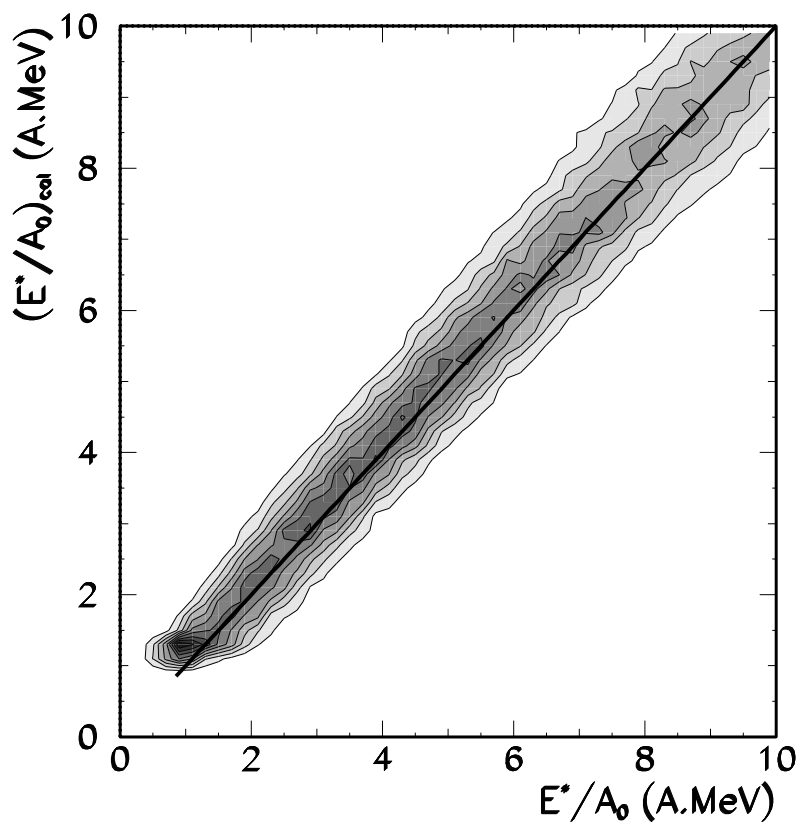

Fig. 7. Correlation between the calorimetric measurement of the excitation energy per nucleon and the input energy of SMM simulations.

The effect of all these approximations is displayed in figure 7, which shows the correlation between the calorimetric excitation energy, calculated with the same uncertainties present in the data, and the input excitation energy of SMM. The average value is reproduced within $0.5 \mathrm{~A} . \mathrm{MeV}$ at the highest excitation energies. The average difference does not exceed $0.2 \mathrm{~A} . \mathrm{MeV}$ in the negative heat capacity region. This fixes the minimum bin width that can 
be used for the data analysis. However an important dispersion around the most probable value is visible. It is important to stress that this width plays a very different role with respect to the freeze out reconstruction uncertainties discussed in the preceding section. In fact, data being analyzed in constant excitation energy bins, the variance of the excitation energy reconstruction does not sum up with the interaction energy variance in eq.(3). The spurious width induced by calorimetry is determined solely by the energy bin, independent of the width of the calorimetric excitation energy distribution of figure 7 . In section 4 we have already shown that the effect of the bin width on $\sigma_{k}$ is not dramatic.

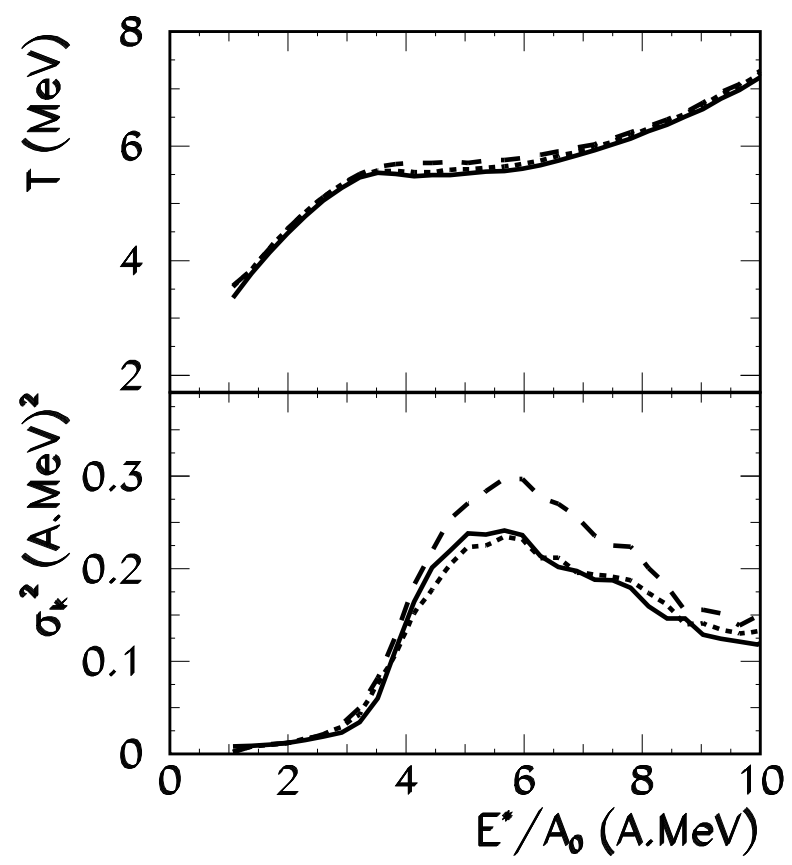

Fig. 8. Effect of calorimetry on the temperature (upper part) and kinetic energy variance (lower part) measurement. Solid lines: freeze out reconstruction as in section 5 and exact excitation energy. Dashed lines: treatment of data as in the experimental sample. Dotted lines: same as dashed, but only events within $10 \%$ from the input energy are retained.

The calorimetric uncertainties, however, can cause an additional spurious effect. A very large spread of the measured excitation energy, if used as a sorting parameter, can lead to event mixing, which can in turn artificially enhance the interaction energy fluctuations. Once again the relative importance of event mixing can be estimated only by a simulation. 
Figure 8 shows the effect of calorimetry on the two ingredients necessary to estimate the heat capacity. The solid lines are the same as the thin solid lines of figure 6 . They give the reference calculation where both $T$ and $\sigma_{k}$ are calculated from the event by event reconstructed $E_{k}$ and the excitation energy per nucleon is the input $E^{*} / A_{0}$ of the simulation. The event mixing due to the imperfect calorimetry (dashed line) does not affect the calculation of average observables like the temperature. On the other side the variance is somewhat enhanced and its functional behavior slightly deformed. If however only events with energy that differs from the input energy less than $10 \%$ are kept, the reference result is approximately recovered (dotted lines). Spurious fluctuations can then be avoided if a constraint is put on the events by means of a conservation law. This idea has already been exploited in section 3 where the doubling of light charged particles was shown not to enhance partial energy fluctuations, if a constraint was put on the size of the reconstructed source. However, because of the calorimetric uncertainty, the total deposited energy is not known a priori. In this case the artificial enhancement of partial energy fluctuations can be minimized if only "conservative" hypothesis are applied to the missing information, as we have already discussed in the previous sections. Every replacement of a not measured quantity by an estimated average value of the same quantity reduces the fluctuations in any observable positively correlated to the experimentally unknown variable. On the other side, if the missing information is replaced with an estimate obtained from measured observables of the same event, this correlation within the same event induces a spurious fluctuation.

This general statement can be better understood by looking at figure 9 which shows the deformation induced by the different unknown quantities one by one. The thick lines give the exact result of the model, while the thin solid lines correspond to the hot freeze out reconstruction as in figure 6 and 8. All the other curves show the effect of the different calorimetric hypotheses which have been applied to the data:

- replacing the (fluctuating) neutron energies with the (average) effective temperature estimate [28] (triangles);

- replacing the (fluctuating) asymptotic masses with the (average) EPAX [27] prescription (open circles);

- doubling the light charged particles and rejecting events that violate mass conservation more than 10\% (crosses).

Finally the squares in figure 9 correspond to a complete calorimetric reconstruction as in the data (all the preceding steps summed up), including also the effect of the MULTICS-MINIBALL filter. A very similar distribution is obtained if the INDRA filter is applied. All these steps do not affect dramatically neither the first nor the second moment of the kinetic energy distribution, showing that the calorimetric spread is well under control. The distortions 


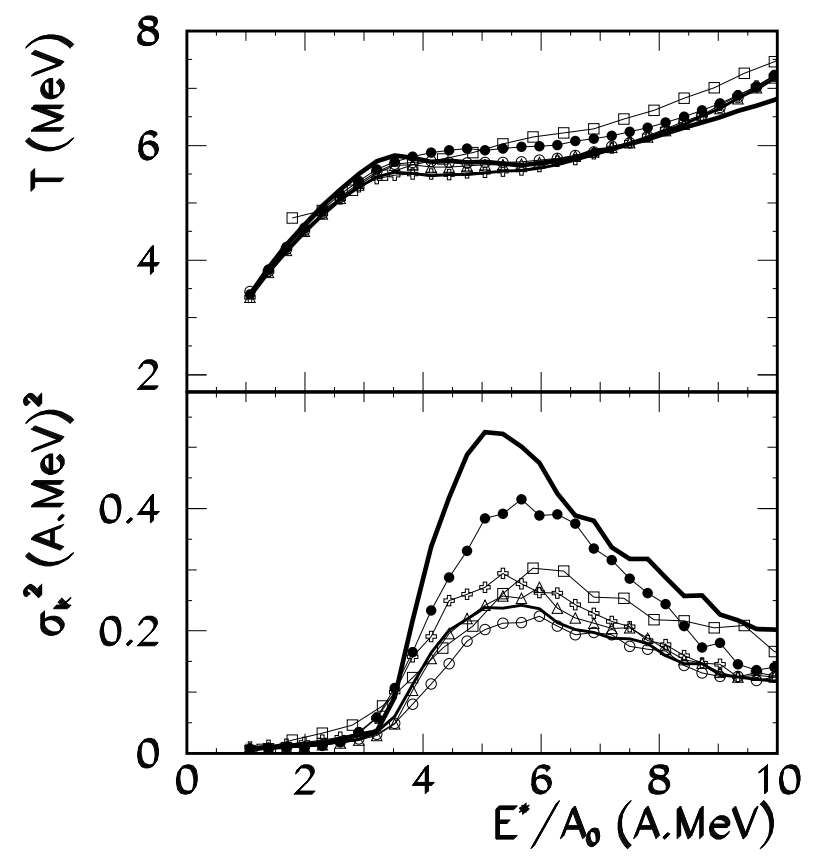

Fig. 9. Effect of the calorimetry on the temperature (upper part) and kinetic energy variance (lower part) measurement. Solid lines: as in figure 6. Symbols: effect of the calorimetric hypotheses one by one (see text).

induced by the filter can be appreciated by comparing the open squares in figure 9 (which correspond to filtered events) to the dashed lines of figure 8 (not filtered events). The main effect of the filter is a general smoothing of the temperature and a slight reduction of fluctuations in the negative heat capacity region.

The only case that produces an evident distortion (full points in figure 9) corresponds to a prescription that attributes to neutrons in each event the same kinetic energy of protons, corrected for the Coulomb barrier. The correlation between protons and neutrons within the same event causes a large calorimetric spread leading to a non negligible event mixing. This is a clear example of an algorithm to be avoided in the analysis of the data. 


\section{The unknown physical parameters: towards a quantitative nu- clear thermodynamics}

In the previous sections we have analyzed the robustness of the fluctuation signal in a simulation where the average values of the observables at freeze-out are known a priori. This is unfortunately not the case for the experimental situation. One can then wonder how much the observation of negative heat capacity in the data depends on the values chosen for these observables. We have already discussed in section 2 the effect of varying the freeze out multiplicity. Switching between the two extreme freeze-out hypotheses leaves the position of the first divergence practically unchanged but strongly modifies the estimated latent heat (see figure 2). The persistence of the negative heat capacity signal may look surprising, knowing that the actual values of the temperature as well as its behavior as a function of the excitation energy are appreciably different in the two freeze out hypotheses (see figure 8 of Ref. [8]). The fluctuation observable $c$ seems in this sense more robust than the caloric curve. This is due to the fact that, even if the (model dependent) temperature in our analysis acts as a normalization factor for the fluctuations (see eq.(2)), the reference fluctuation scale $\left(c_{k}\right)$ and the fluctuation itself $\left(\sigma_{k}\right)$ are consistently derived from the same equation of state. Once the multiplicity is fixed, the actual freeze-out composition i.e. the precise relationship between mass and charge of the primary fragments does not modify the results as discussed in section 6 .

A parameter to be discussed is the freeze-out volume, which determines the average value of the Coulomb potential energy. As already remarked in section 6 , the Coulomb energy is positively correlated to the fragment multiplicity implying that the effect of the volume will depend on the freeze-out hypothesis. If we change in an arbitrary way the freeze out volume as well as the other characteristics of the freeze-out configuration, the quantitative result for the heat capacity varies considerably, though only very extreme and unrealistic hypotheses suppress the negative branch [24]. However it is possible to make a few steps further. In fact the average volume can be experimentally estimated in a quite precise way from the mean detected fragment kinetic energy $\left(<E_{k i n}>\right)$ of the data sample under study. Concerning the QP data, the measured fragment energy distributions are compatible with a collective (i.e non thermal, non Coulomb) component at most $0.7 \mathrm{~A} . \mathrm{MeV}$ at $7 \mathrm{~A} . \mathrm{Mev}$ of excitation energy, and about $0.4 \mathrm{~A} \cdot \mathrm{MeV}$ at $\mathrm{E}^{*}=4 \mathrm{~A} \cdot \mathrm{MeV}$ [8]. This component has been subtracted from the presented data and all the results presented in this paper would not change in any sizeable way if this component was kept. Therefore for this data sample one can perform a many body Coulomb trajectory calculation, by randomly placing the reconstructed primary fragments in a spherical volume and letting them evolve in the Coulomb field. Under the hypothesis that, on average, light charged particle evaporation does not 


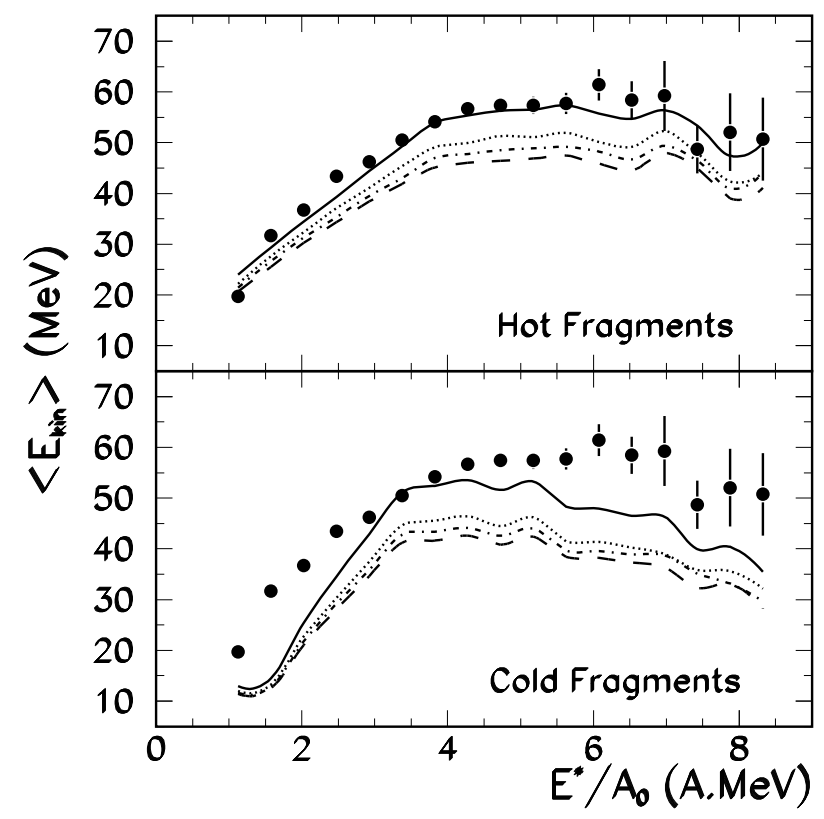

Fig. 10. Average fragment $(Z \geq 3)$ kinetic energy as a function of the excitation energy for QP data. Symbols: rough experimental data; bars represent the statistical errors. Lines: many body Coulomb trajectory calculations for a volume of $\sim 3 V_{0}$ (full), $4 V_{0}$ (dotted), $5 V_{0}$ (dashed dotted) and $6 V_{0}$ (long dashed) within the two extreme freeze out hypotheses.

affect fragment velocities, the superposition of the average Coulomb and thermal motion provides an observable directly comparable with $<E_{k i n}>$. This comparison should allow to select directly from data a (possibly energy dependent) range of freeze-out volumes. The result is displayed in figure 10 for the quasi-projectile data in the two extreme freeze out hypotheses. The full lines correspond to the smallest volumes that contain the fragments. This minimum volume turns out to be on average $\sim 3 V_{0}$. For each bin energy the temperature, determining the average thermal motion $1.5 \cdot T$, is univocally determined from energy conservation, using the kinetic equation of state eq.(4). Of course the actual value of the temperature depends also on the level density parameter entering equation (4). This extra source of uncertainty however does not modify in any sizeable way the results. In fact the Coulomb contribution by far dominates the average kinetic energies, implying that the average freeze out volume can be estimated independently of the level density parameter.

Once the freeze out hypothesis is fixed, the results of figure 10 define unambiguously the average volume. From this comparison the cold fragment hypothesis is ruled out, because the average Coulomb energy per fragment turns out much smaller than in the data. We note by passing that the good reproduc- 
tion of the measured average kinetic energies can be considered an additional evidence of the equilibration of the data sample. Any collective component or dynamical mechanism in fragment formation would lead to a deviation between the detected energies and the ones reconstructed through Coulomb trajectories; this discrepancy would increase with increasing deposited energy.

The situation is slightly more complicated in the case of central $X e+S n$ collisions. In this case $\left\langle E_{k i n}>\right.$ gives a measure of the Coulomb repulsion plus the radial collective flow. In the hot fragment hypothesis a volume of $3 V_{0}$ is consistent with experimental data if a collective flow of $0.6 \mathrm{~A} . \mathrm{MeV}$ is assumed, but a volume as large as $6 V_{0}$ can still reproduce the fragment kinetic energy if the collective flow is $1 \mathrm{~A} . \mathrm{MeV}[2]$ for $32 \mathrm{~A} . \mathrm{MeV}$ bombarding energy. One could also consider smaller freeze out volumes and lower collective components, as suggested by some theoretical speculations [29].

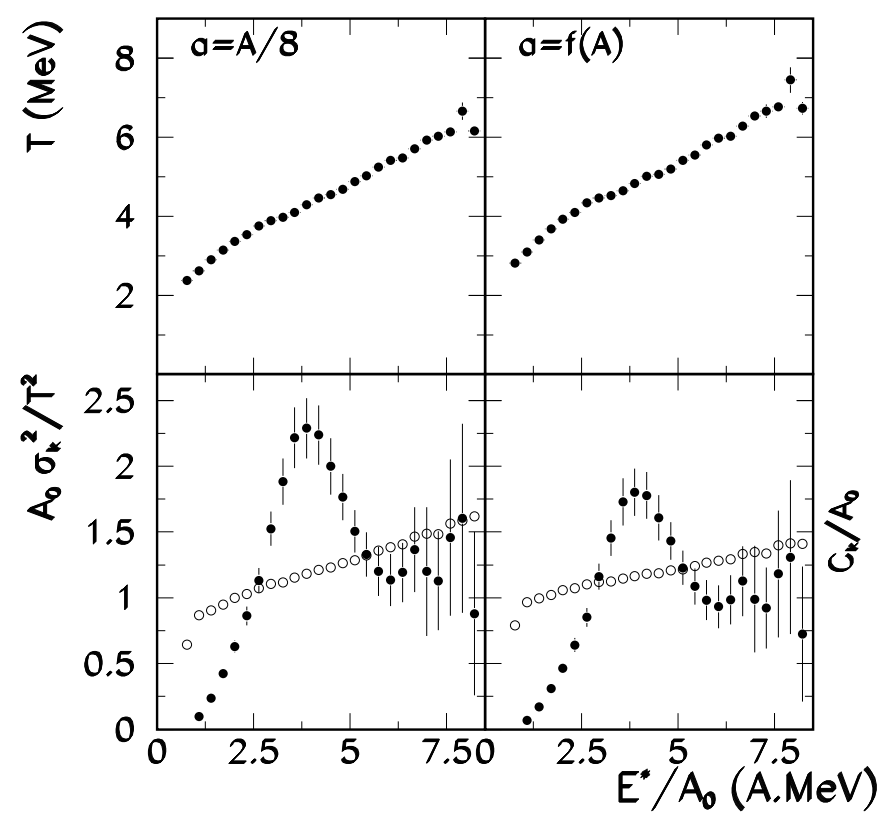

Fig. 11. Temperature, kinetic energy fluctuation and kinetic heat capacity for the $Q P$ data in the hot fragment hypothesis (freeze-out volume $\sim 3 V_{0}$ with two different prescriptions for the level density parameter (for $f(A)$ see Ref. [26]).

The last unknown entering the total heat capacity eq.(2) is the level density parameter $a$ that determines the freeze out temperature. The effect of changing $a$ is illustrated in figure 11 for the quasi-projectile data: the net effect is an uncertainty in the estimate of the temperature that never exceeds about 0.5 $\mathrm{MeV}$ per nucleon. If the abnormal fluctuation signal is clearly independent of the detailed structure of the level density parameter, the localization of the 
divergences however depends on the value assumed. It is important to keep in mind that in any case the latent heat cannot be quantitatively estimated, because of the fluctuation suppression operated by our reconstruction method. This means that the distance between the two crossing points between $\sigma_{k}^{2} / T^{2}$ and $c_{k}$ in figure 11 represents in both cases a lower limit for the actual value of the latent heat.
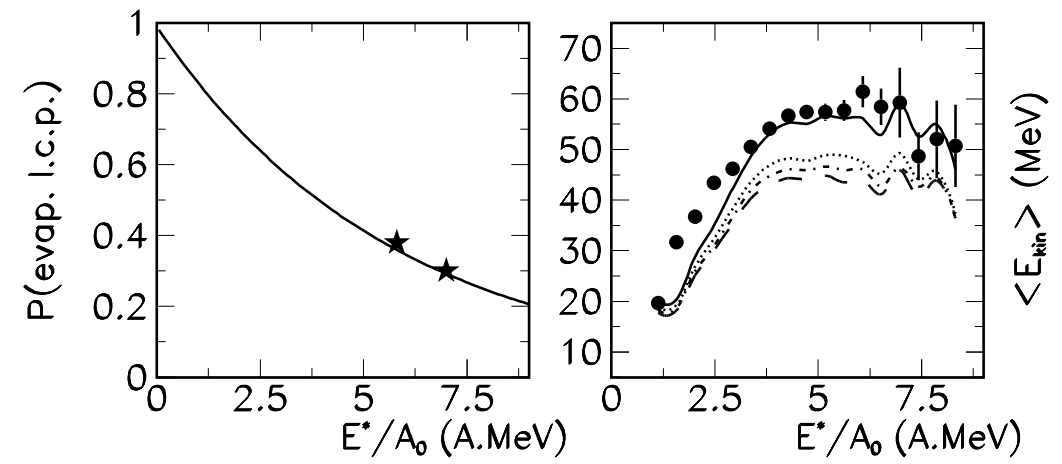

Fig. 12. Left part: percentage of evaporated light charged particles as a function of the excitation energy; symbols: experimental data from Ref. [30]; line: exponential extrapolation. Right part: as figure 10, but lines refer to the freeze out reconstruction hypothesis defined in the left panel.

The study of the measured $<E_{k i n}>$ has allowed us to exclude the cold fragment hypothesis for the reconstruction of the freeze out. However the hot fragment hypothesis is also an extreme scenario for the secondary deexcitation. It is reasonable to expect that the percentage of primary light particles is neither $0 \%$ nor $100 \%$ and rather depends on the deposited energy. A more quantitative insight into the thermodynamical properties of the hot nuclear systems can be achieved if experimental information about the freeze out composition is inserted in the freeze-out reconstruction. An example is given by Ref. [30]. Velocity correlations between fragments and light charged particles allow to estimate the percentage of secondarily evaporated particles (symbols in left panel of Fig.12) and the average excitation energy of primary fragments (stars in the right panel of Fig.13). This work [30] has been performed on the same $X e+S n$ sample considered in the present paper. We have already mentioned that the size of the sources measured in central $X e+S n$ and in the $A u+A u$ peripheral events are very similar. If we assume that for a given excitation energy the breakup of the sources does not depend on the entrance channel, we can perform for the quasi-projectile events a freeze-out reconstruction based on the results of Ref. [30].

Within this freeze out assumption it is possible to reproduce the QP measured 
kinetic energies as in figure 10 with a freeze out volume $\sim 3 V_{0}$ (corresponding to the minimum volume that contains the fragments). This is shown in the right part of figure 12 . The $\left\langle E_{k i n}>\right.$ observable is quite well reproduced by the superposition of Coulomb and thermal motion. Only at energies lower than about $3 \mathrm{~A} . \mathrm{MeV}$ the kinetic energies are underestimated. This is clearly due to the arbitrariness of our low energy extrapolation. While it is reasonable to assume that the totality of light charged particles is evaporated at an excitation energy close to zero, a comparison between figure 12 and figure 10 suggests that the percentage of evaporated light charged particles in the interval $0<E^{*} / A_{0}<3 \mathrm{~A} . \mathrm{MeV}$ should be less steep. Some data in this energy domain are clearly needed to better constrain the curve.
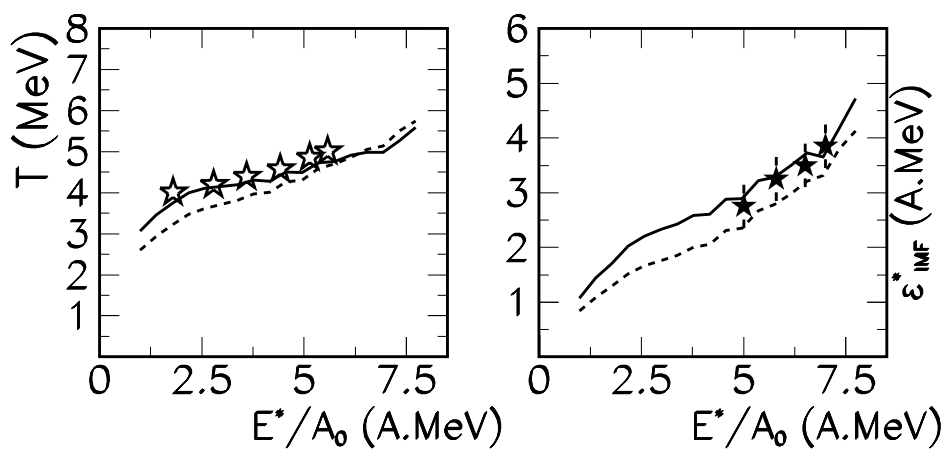

Fig. 13. Left: temperature measured with an isotopic thermometer [19] (open symbols) and from eq.(4) for the QP (lines). Right: primary fragment internal excitation energy measured from velocity correlation measurements in central collisions [30] (full symbols) and from eq.(4) for the QP (lines). Dashed lines correspond to a level density parameter $a=A / 8$ while $a=f(A)$ (see Ref. [26]) is taken for the full lines.

The last quantity to be settled in the heat capacity analysis is the level density parameter. This can be fixed by injecting as much experimental information as possible from independent measurements. As an example, symbols in the left panel of figure 13 show the isotopic temperatures from the Carbon thermometer measured for the quasi projectile data [19]. For this specific thermometer side feeding effects have been estimated to induce an uncertainty of about 0.5 $\mathrm{MeV}$ at most [19]. Symbols in the right panel of figure 13 show the internal excitation energy of primary fragments, experimentally reconstructed for the central $X e+S n$ data in Ref. [30]. Both sets of data are compared in figure 13 with the kinetic thermometer eq.(4) measured for the quasi projectile data sample. The results of figure 13 do not allow to discriminate between the two different prescriptions for the level density parameter, however they indicate that the temperature cannot vary more than what shown by figure 11 . 


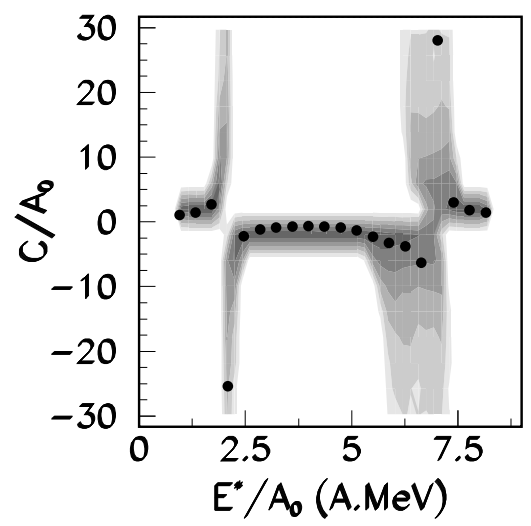

Fig. 14. Heat capacity per nucleon as a function of the excitation energy for the $Q P$ system with the freeze out reconstruction constrained to reproduce the experimental values of Ref. [30]. A level density $a=A / 8$ has been assumed.
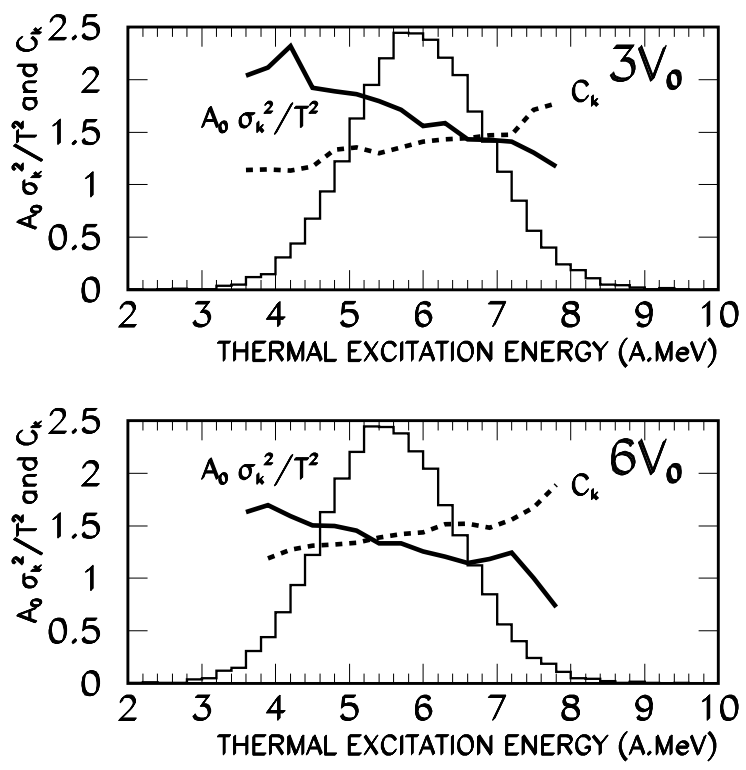

Fig. 15. Normalized fluctuations (full lines), kinetic heat capacity (dashed lines) and excitation energy distribution (histograms) for the central 32 A.MeV Xe+Sn system at the lowest and highest freeze out volume with the freeze out reconstruction constrained to reproduce the experimental values of Ref. [30]. A level density $a=A / 8$ has been assumed.

The final result for the heat capacity measured in the two Au-like systems is presented in figures 14 and 15. In both cases the freeze out volume and multiplicity have been fixed through the independent experimental constraints shown in figures 12 and 13. In the case of central collisions (figure 15) only 
a limiting range of possible freeze out volumes $\left(3 V_{0}<V<6 V_{0}\right)$ has been estimated from the analysis of asimptotic $\left\langle E_{k i n}>\right.$, because of the additional uncertainty coming from the radial collective flow [2,31]. The compatibility of the two sets of data and the presence of divergences and a negative branch for $c$ is indisputable.

\section{Conclusions}

The sources of uncertainty that arise in the thermostatistical analysis of well detected multifragmenting nuclear systems have been analyzed. In order to be able to study mean values as well as higher moments of the distributions, the quality of the calorimetric reconstruction is essential. A general protocol has been established to deal with missing information in such a way that the distortions due to an imperfect detection are minimized. The presence of a first order liquid-to-gas-like phase transition in nuclear multifragmentation $[1,2]$ is confirmed by this analysis. The negative heat capacity signal survives to all the uncertainties due to the different reconstruction hypotheses. A negative value for the heat capacity is signed by abnormally large interaction energy fluctuations. In order to disentangle between physical fluctuations and experimental uncertainties we have systematically adopted a procedure which suppresses the variance of all not measured quantities. The challenge for the next future is to reintroduce this missing fluctuation by more complete measurements and minimum bias simulations. A promising technique to recover the missing information is suggested by figure 10 above. More sophisticated Coulomb trajectories can be employed to compare the variance of the asymptotic fragment kinetic energy $\left(<E_{k i n}>\right)$ distribution. Fluctuations at freeze out could then be tuned to reproduce this observable [32].

The ultimate challenge of these analysis is the reconstruction of the nuclear phase diagram as a function of mass and possibly isospin. To this aim as accurate as possible measurements of relevant thermodynamical parameters at freeze out are essential. A lot of work in this direction has been already done and will hopefully continue and become more precise with next generation detectors [33]. With only two independent measurements (for instance temperature and volume) at the $10 \%$ or $20 \%$ level, almost all ambiguities in the quantitative estimate of the heat capacity is removed. Any other independent observation of freeze out variables can then be used as a cross check of the consistency of the procedure. 


\section{Acknowledgements}

This work has been partially supported by NATO grants CLG 976861 and by grants of the Italian Ministry of University and Scientific and Technological Research under grants Cofin99.

\section{References}

[1] M. D'Agostino et al., Phys. Lett. B 473 (2000) 219.

[2] N. Le Neindre et al., contribution to the XXXVIII Winter Meeting on Nucl. Phys., Bormio (Italy), Ed. I. Iori and A. Moroni, (2000) p.404 and to be published.

[3] W. Thirring, Zeit. Phys. 235 (1970) 339;

D. Lynden-Bell, Proc. of the XXth IUPAP Int.Conf on Stat. Phys., Paris, July 20-24 (1998) and cond-mat/9812172.

[4] D. H. E. Gross, Phys. Rep. 279 (1997) 119; D.H.E. Gross and E. Votyakov, Europhys. Journ. B15 (2000) 115.

[5] F. Gulminelli and P. Chomaz, Phys. Rev. Lett. 82 (1999) 1402.

[6] M. Schmidt et al., Phys. Rev. Lett. 79 (1997) 99; Nature 393, 238 (1998); Phys. Rev. Lett. 86 (2001) 1191.

[7] P. Chomaz and F. Gulminelli, Nucl. Phys. A647 (1999) 153.

[8] M. D’Agostino et al., Nucl. Phys. A650 (1999) 329.

[9] N. Marie et al., Phys. Lett. B391 (1997) 15.

[10] J. Richert and P. Wagner, arXiv:nucl-th/0009023 v2 and to be published in Phys. Rep.

[11] R.Botet, M. Ploszajczak, Phys. Rev. E62 (2000) 1825 and nucl-ex/0101012.

[12] J. M. Carmona, N. Michel, J. Richert and P. Wagner, Phys. Rev. C61 (2000) 037304.

[13] J. L. Lebowitz et al., Phys.Rev. 153 (1967) 250.

[14] Ph. Chomaz, F. Gulminelli and V. Duflot, Phys. Rev. Lett. 85 (2000)3587.

[15] J. Cugnon and D. L'Hote, Nucl. Phys. A397 (1983) 519.

[16] J. D. Frankland et al., contribution to the XXXV Winter Meeting on Nucl. Phys., Bormio (Italy), Ed. I. Iori, (1997) p.323.

[17] J. F. Lecolley et al., Nucl. Instr. and Methods A441 (2000) 517. 
[18] C. P. Montoya et al., Phys. Rev. Lett. 73 (1994) 3070;

T. Lefort et al., Nucl. Phys. A662 (2000) 397;

E. Plagnol et al., Phys. Rev. C61 (2000) 014606.

[19] P. M. Milazzo et al., Phys. Rev. C58 (1998) 953.

[20] J. P. Bondorf, A. S. Botvina, A. S. Iljinov, I. N. Mishustin, K. Sneppen, Phys. Rep. 257 (1995) 133.

[21] J. B. Elliot and A. S. Hirsch, Phys. Rev. C61 (2000) 054605.

[22] R. J. Charity et al., Nucl. Phys. A483 (1988) 371.

[23] J. P. Bondorf, A. S. Botvina, I. N. Mishustin, Phys. Rev. 58C (1998) R27.

[24] M. D'Agostino et al., contribution to the XXXVIII Winter Meeting on Nucl. Phys., Bormio (Italy), Ed. I. Iori and A. Moroni, (2000) p.386.

[25] P. Désesquelles, M. D’Agostino, A. S. Botvina, M. Bruno et al., Nucl. Phys. A633 (1998) 547.

[26] The level density parameter is assumed, as in Ref. [20], continuously varying from $A / 8$ for the lightest nucleus $A=7$ to $A / 12$ for the heaviest one $A=197$, to take into account different surface and volume contributions.

[27] K. Sümmerer, W. Bruüchle, D. J. Morrissey, M. Schädel, B. Szweryn and Yang Weifan, Phys. Rev. C 42 (1990) 2546.

[28] J. A. Hauger, P. Warren, S. Albergo, F. Bieser and EOS collaboration, Phys. Rev. C 57 (1998) 764 and references quoted therein.

[29] X. Campi et al., Nucl. Phys. A681 (2001) 458c;

A. Chernomoretz, M. Ison, S. Ortiz, C. Dorso, nucl-th/0101061, 26 Jan. 2001.

[30] N. Marie, A. Chbihi, J. B. Natowitz, A. Le Fèvre et al., Phys. Rev. C58 (1998) 256 ;

S. Hudan et al., contribution to the XXXVIII Winter Meeting on Nucl. Phys., Bormio (Italy), Ed. I. Iori, (2000) p.404.

[31] N. Le Neindre, These de l'Université de Caen (1999) unpublished.

[32] M. D'Agostino et al., in preparation.

[33] A. Pagano et al. Nucl. Phys. A681 (2001) 331c. 